\title{
Interrelationship between Different Seed Quality Parameters in Fennel
}

\author{
Deepak Kumar Singhal*, Vinod Kumar, S.K. Tehlan, Pooja Rani and Amit Kumar
}

Department of Vegetable Science, CCS Haryana Agricultural University, Hisar- 125004, India

\author{
*Corresponding author
}

\section{A B S T R A C T}

\begin{tabular}{|l|}
\hline Key w o r d s \\
Ageing, seed lots, \\
seed quality, \\
correlation, field \\
emergence (\%), \\
coriander.
\end{tabular}

The present investigation was carried out on fennel seeds of fifteen genotypes viz., HF 33, HF 101, HF 102, HF 103, HF 104, HF 105, HF 106, HF 107, HF 108, HF 109, HF 114, HF 115, HF 118, HF 122 and HF 124 with three lots of seed viz., freshly harvested seed (Lot-1), one year old seed (Lot-2) and two year old seed (Lot-3). The seeds were subjected to study the effect of ambient storage on different seed viability and vigour parameters. Correlation coefficient analysis was employed to find out the interrelationship between various seed quality parameter viz., test weight, standard germination, seedling length, seedling fresh weight, seedling dry weight, seedling vigour index-I, seedling vigour index- II, accelerated ageing test, Tetrazolium test, dehydrogenase activity test, electrical conductivity test, $\mathrm{pH}$ exudates test and field emergence (\%). Field emergence showed positive and significant correlation with seedling length (0.839), standard germination (0.879), seedling fresh weight $(0.808)$, seedling dry weight $(0.742)$, vigour index-I (0.880), vigour index-II (0.772), tetrazolium test $(0.890)$, accelerated ageing test (0.729), dehydrogenase activity test (0.757) and $\mathrm{pH}$ exudates test $(0.734)$ while negative and significant correlation was found with electrical conductivity (0.845). As standard germination, seedling vigour index-I and tetrazolium test were found highly correlated with field emergence they can be used as reliable predictors of field emergence $(\%)$.

\section{Introduction}

Fennel (Foeniculum vulgare Mill.) which belongs to the family Apiaceae is one of the widely cultivated seed spice in temperate and sub tropical regions of the world. A diet with desired quantity of fennel could bring potential health benefits due to its valuable nutritional composition with respect to presence of essential fatty acids (Barros et al., 2010).

In recent years, increased interests in improvement of agricultural yield of fennel due to its medicinal properties and essential oil content has encouraged cultivation of the plant on large scale. High quality seed is the key to successful agriculture. Modern agriculture with its favouritism for technology and precision, demands that each and every seed should readily germinate and produce a healthy seedling to ensure high yield. Uniformity of growth and synchrony in development are highly desirable characters for mechanized cultural operations. As such, only high quality i.e., genetically pure and morphologically, pathologically and physiologically sound seed is capable of increasing the productivity. The seeds should also have better storability to produce good 
crop during the next season. The laboratory germination test provides information about the seedling emergence potential of seed lots under favourable conditions (Perry, 1978). In the present study attempt has been made to evaluate and compare various seed quality tests with a view to identify dependable tests for seed testing laboratories to know the planting value of seeds

\section{Materials and Methods}

The present study was carried out on fennel seeds of fifteen genotypes viz., HF 33, HF 101, HF 102, HF 103, HF 104, HF 105, HF 106, HF 107, HF 108, HF 109, HF 114, HF 115, HF 118, HF 122 and HF 124 with three lots of seed viz., freshly harvested seed $\left(\operatorname{Lot}_{1}\right)$, one year old seed $\left(\operatorname{Lot}_{2}\right)$ and two year old seed $\left(\mathrm{Lot}_{3}\right)$ collected from Department of Vegetable Science, CCS H.A.U, Hisar during 2014-15. All the seed lots were stored under ambient condition analyzed for test weight, standard germination test (\%), seedling length (cm), seedling fresh weight, seedling dry weight (mg), seedling vigour index-I, seedling vigour index-II, accelerated ageing test (\%), tetrazolium test (viability \%), $\mathrm{pH}$ exudate test (\%), dehydogenase activity test (OD g $\left.\mathrm{ml}^{-1}\right)$, electrical conductivity test $\left(\mu \mathrm{S} \mathrm{cm} \mathrm{cm}^{-1} \mathrm{seed}^{-1}\right)$ and field emergence (\%) in seed testing laboratory, Department of Seed Science and Technology, CCS Haryana Agricultural University.

The experiment was conducted in a Completely Randomized Design for laboratory parameters and, for field parameters; the same was designed in Randomized Block. The angular transformation was applied to the percent data and the transformed data were subjected to the statistical analysis on the basis of the model described by Ostle and Mensing (1975). The correlation coefficient between various seed viability and vigour parameters in laboratory and field emergence was carried out as per standard procedure given below:

$$
r=\frac{\text { Cov. }(X Y)}{\sqrt{\text { Variance of } X \times \text { Variance of } Y}}
$$

Where,

$\mathrm{r}=$ Correlation coefficient

$\operatorname{Cov}(\mathrm{x}, \mathrm{y})=$ Covariance between characters $\mathrm{x}$ and $\mathrm{y}$

\section{Results and Discussion}

The correlation between field and laboratory parameters of freshly harvested seed lot is given in Table 1. Standard germination is positively and significantly correlated with test weight (0.849) seedling length (0.910), seedling fresh weight $(0.829)$, vigour index-I (0.968), vigour index-II (0.940), tetrazolium test (0.907), accelerated ageing test (0.548), dehydrogenase activity test (0.853), $\mathrm{pH}$ exudates test (0.849) and field emergence (0.974) while negatively and significant correlation was found with electrical conductivity (0.972). Field emergence showed positive and significant correlation with test weight (0.843) seedling length (0.930), seedling fresh weight (0.807), vigour index-I (0.971), vigour index-II (0.883), tetrazolium test (0.882), accelerated ageing test (0.543), dehydrogenase activity test $(0.867), \mathrm{pH}$ exudates $(0.809)$ and negative and significant correlation with electrical conductivity (0.976). Correlation studies revealed highly significant and positive correlation between standard germination, $\mathrm{pH}$ exudate and field emergence, suggesting that these tests can be used as reliable predictor of field emergence of fennel seeds. The results obtained by Ram et al., (1991) support this finding, that vigour indices showed positive significant association with field emergence in pigeon pea. Similar results were also reported in coriander (Kumar et al., 2015) and in fenugreek (Kumari et al., 2014). 
Table.1 Interrelationship between different laboratory parameters of seed quality and field emergence (\%) of freshly harvested seed of fennel

\begin{tabular}{|c|c|c|c|c|c|c|c|c|c|c|c|c|c|}
\hline Parameters & TW & SL & SG & SFW & SDW & VI-I & VI-II & $\mathbf{T Z}$ & $\mathbf{A A T}$ & DHA & $\mathbf{E C}$ & pH & FE $(\%)$ \\
\hline \multicolumn{14}{|l|}{ TW } \\
\hline $\mathrm{SL}$ & $0.863 * *$ & & & & & & & & & & & & \\
\hline SG & $0.849 * *$ & $0.910^{* *}$ & & & & & & & & & & & \\
\hline SFW & $0.713 * *$ & $0.762 * *$ & $0.829 * *$ & & & & & & & & & & \\
\hline SDW & $0.720 * *$ & $0.718^{* *}$ & $0.873^{* *}$ & $0.738 * *$ & & & & & & & & & \\
\hline VI-I & $0.885^{* *}$ & $0.984 * *$ & $0.968 * *$ & $0.810 * *$ & $0.794 * *$ & & & & & & & & \\
\hline VI-II & $0.789 * *$ & $0.797^{* *}$ & $0.940^{* *}$ & $0.793 * *$ & $0.987 * *$ & $0.872 * *$ & & & & & & & \\
\hline $\mathrm{TZ}$ & $0.801 * *$ & $0.863 * *$ & 0.907 ** & $0.609 * *$ & $0.730 * *$ & $0.897 * *$ & $0.804 * *$ & & & & & & \\
\hline AAT & $0.658 * *$ & $0.610 * *$ & $0.548 *$ & 0.475 & 0.349 & $0.604 *$ & 0.426 & $0.519^{*}$ & & & & & \\
\hline DHA & $0.870^{* *}$ & $0.888 * *$ & $0.853^{* *}$ & $0.608^{* *}$ & $0.700 * *$ & $0.900 * *$ & $0.772 * *$ & $0.804 * *$ & $0.697 * *$ & & & & \\
\hline $\mathrm{EC}$ & $-0.880 * *$ & $-0.924 * *$ & $-0.972 * *$ & $-0.823 * *$ & $-0.831 * *$ & $-0.966 * *$ & $-0.901 * *$ & $-0.880 * *$ & $-0.525^{*}$ & $-0.834 * *$ & & & \\
\hline $\mathrm{pH}$ & $0.803 * *$ & $0.823 * *$ & $0.849 * *$ & $0.843 * *$ & $0.731^{* *}$ & $0.854^{* *}$ & $0.791^{* *}$ & $0.718^{* *}$ & $0.739 * *$ & $0.812 * *$ & $-0.826 * *$ & & \\
\hline $\mathrm{FE}(\%)$ & $0.843 * *$ & $0.930 * *$ & $0.974 * *$ & $0.807 * *$ & $0.805^{* *}$ & $0.971 * *$ & $0.883^{* *}$ & $0.882^{* *}$ & $0.543^{*}$ & $0.867 * *$ & $-0.976^{* *}$ & $0.809^{* *}$ & \\
\hline
\end{tabular}

$*$ Significant at $5 \%(\mathrm{p}=0.05)$

** Significant at $1 \%(\mathrm{p}=0.01)$

TW = Test Weight, SL = Seedling length, SG =standard germination, SFW = Seedling fresh weight, SDW = Seedling dry weight, VI-I = Vigour index-I, VI-II $=$ Vigour index-II, TZ $=$ Tetrazolium test, $\mathrm{AA}=$ Accelerated ageing test, $\mathrm{DHA}=$ Dehydrogenase activity test, $\mathrm{EC}=$

Electrical conductivity test, $\mathrm{pH}=\mathrm{pH}$ exudates test, $\mathrm{FE}=$ Field emergence. 
Table.2 Interrelationship between different laboratory parameters of seed quality and field emergence (\%) of one year stored seed of fennel

\begin{tabular}{|c|c|c|c|c|c|c|c|c|c|c|c|c|c|}
\hline Parameters & TW & SL & SG & SFW & SDW & VI-I & VI-II & $\mathbf{T Z}$ & AAT & DHA & EC & pH & FE $(\%)$ \\
\hline \multicolumn{14}{|l|}{$\mathrm{TW}$} \\
\hline $\mathrm{SL}$ & $0.750^{* *}$ & & & & & & & & & & & & \\
\hline SG & $0.737^{* *}$ & $0.794^{* *}$ & & & & & & & & & & & \\
\hline SFW & $0.720^{* *}$ & $0.769^{* *}$ & $0.739^{* *}$ & & & & & & & & & & \\
\hline SDW & $0.817^{* *}$ & $0.918^{* *}$ & $0.889^{* * *}$ & $0.820^{* *}$ & & & & & & & & & \\
\hline VI-I & $0.789^{* *}$ & $0.976^{* *}$ & $0.907^{* *}$ & $0.799^{* *}$ & $0.952^{* *}$ & & & & & & & & \\
\hline VI-II & $0.824^{* *}$ & $0.908^{* *}$ & $0.931^{* *}$ & $0.822^{* * *}$ & $0.994^{* *}$ & $0.961^{* *}$ & & & & & & & \\
\hline $\mathrm{TZ}$ & $0.722^{* *}$ & $0.785^{* *}$ & $0.931^{* *}$ & $0.708^{* *}$ & $0.794^{* *}$ & $0.874^{* * *}$ & $0.839^{* *}$ & & & & & & \\
\hline AAT & $0.692^{* *}$ & $0.618^{* *}$ & $0.607^{* *}$ & $0.626^{* *}$ & $0.562^{*}$ & $0.653^{* *}$ & $0.590^{*}$ & $0.758^{* *}$ & & & & & \\
\hline DHA & $0.859^{* * *}$ & $0.675^{* *}$ & $0.747^{* *}$ & $0.693^{* *}$ & $0.759^{* * *}$ & $0.735^{* *}$ & $0.773^{* *}$ & $0.790^{* *}$ & $0.739^{* *}$ & & & & \\
\hline EC & $-0.587^{*}$ & $-0.775^{* * *}$ & $-0.787^{* *}$ & $-0.720^{* *}$ & $-0.867^{* *}$ & $-0.816^{* *}$ & $-0.864^{* * *}$ & $-0.683^{* * *}$ & -0.439 & $-0.640^{* *}$ & & & \\
\hline $\mathrm{pH}$ & $0.762^{* *}$ & $0.749^{* * *}$ & $0.772^{* *}$ & $0.865^{* *}$ & $0.775^{* *}$ & $0.800^{* *}$ & $0.796^{* *}$ & $0.753^{* *}$ & $0.633^{* *}$ & $0.657^{* *}$ & $-0.588^{*}$ & & \\
\hline $\mathrm{FE}(\%)$ & $0.795^{* *}$ & $0.804^{* *}$ & $0.805^{* *}$ & $0.739^{* *}$ & $0.819^{* *}$ & $0.850^{* * *}$ & $0.837^{* *}$ & $0.834^{* *}$ & $0.786^{* *}$ & $0.720^{* *}$ & $-0.729^{* *}$ & $0.706^{* *}$ & \\
\hline
\end{tabular}

*Significant at $5 \%(\mathrm{p}=0.05)$

** Significant at $1 \%(\mathrm{p}=0.01)$

$\mathrm{TW}=$ Test Weight, $\mathrm{SL}=$ Seedling length, $\mathrm{SG}=$ standard germination, SFW = Seedling fresh weight, SDW = Seedling dry weight, VI-I = Vigour index-I, VI-II = Vigour index-II, TZ = Tetrazolium test, AA = Accelerated ageing test, DHA = Dehydrogenase activity test, EC =

Electrical conductivity test, $\mathrm{pH}=\mathrm{pH}$ exudates test, $\mathrm{FE}=$ Field emergence. 
Table.3 Interrelationship between different laboratory parameters of seed quality and field emergence (\%) of two year stored seeds of fennel

\begin{tabular}{|c|c|c|c|c|c|c|c|c|c|c|c|c|c|}
\hline Parameters & TW & SL & SG & SFW & SDW & VI-I & VI-II & $\mathbf{T Z}$ & AAT & DHA & EC & pH & FE $(\%)$ \\
\hline \multicolumn{14}{|l|}{$\mathrm{TW}$} \\
\hline SL & 0.441 & & & & & & & & & & & & \\
\hline SG & $0.613^{\text {*** }}$ & $0.702^{* *}$ & & & & & & & & & & & \\
\hline SFW & 0.413 & $0.782^{* *}$ & $0.685^{* * *}$ & & & & & & & & & & \\
\hline SDW & 0.433 & $0.958^{* *}$ & $0.611^{* *}$ & $0.794^{* *}$ & & & & & & & & & \\
\hline VI-I & $0.599^{*}$ & $0.917^{* *}$ & $0.926^{* *}$ & $0.787^{\text {** }}$ & $0.850^{* *}$ & & & & & & & & \\
\hline VI-II & $0.562^{*}$ & $0.956^{* *}$ & $0.791^{* *}$ & $0.815^{\text {*** }}$ & $0.965^{* * *}$ & $0.950^{* * *}$ & & & & & & & \\
\hline $\mathrm{TZ}$ & 0.373 & $0.820^{* *}$ & $0.822^{* *}$ & $0.795^{* *}$ & $0.769^{* *}$ & $0.881^{* *}$ & $0.842^{* *}$ & & & & & & \\
\hline AAT & $0.649^{\text {*** }}$ & $0.617^{* *}$ & $0.879^{* * *}$ & $0.621^{\text {** }}$ & $0.545^{*}$ & $0.817^{* *}$ & $0.705^{\text {*** }}$ & $0.779^{* *}$ & & & & & \\
\hline DHA & 0.378 & $0.732^{* *}$ & $0.717^{* *}$ & $0.633^{* *}$ & $0.718^{* * *}$ & $0.781^{* *}$ & $0.774^{* *}$ & $0.768^{* *}$ & $0.793^{* *}$ & & & & \\
\hline $\mathrm{EC}$ & -0.226 & -0.032 & -0.364 & -0.273 & 0.004 & -0.221 & -0.125 & -0.229 & -0.462 & -0.099 & & & \\
\hline $\mathrm{pH}$ & 0.290 & $0.608^{* *}$ & $0.627^{* *}$ & $0.670^{* * *}$ & $0.489^{*}$ & $0.655^{* *}$ & $0.558^{*}$ & $0.612^{* *}$ & $0.640^{* * *}$ & $0.581^{*}$ & -0.471 & & \\
\hline $\mathrm{FE}(\%)$ & 0.295 & $0.570^{*}$ & $0.540^{*}$ & $0.544^{*}$ & $0.488^{*}$ & $0.603^{*}$ & $0.553^{*}$ & $0.623^{* *}$ & $0.526^{*}$ & 0.420 & -0.374 & 0.314 & \\
\hline
\end{tabular}

$*$ Significant at $5 \%(\mathrm{p}=0.05)$

** Significant at $1 \%(\mathrm{p}=0.01)$

TW = Test Weight, SL = Seedling length, SG =standard germination, SFW = Seedling fresh weight, SDW = Seedling dry weight, VI-I = Vigour index-I, VI-II = Vigour index-II, TZ = Tetrazolium test, $\mathrm{AA}=$ Accelerated ageing test, $\mathrm{DHA}=$ Dehydrogenase activity test, $\mathrm{EC}=$

Electrical conductivity test, $\mathrm{pH}=\mathrm{pH}$ exudates test, $\mathrm{FE}=$ Field emergence. 
Table.4 Interrelationship between different laboratory parameters of seed quality and field emergence (\%) of means of naturally aged seed of fennel

\begin{tabular}{|c|c|c|c|c|c|c|c|c|c|c|c|c|c|}
\hline Parameters & TW & SL & SG & SFW & SDW & VI-I & VI-II & $\mathbf{T Z}$ & AAT & DHA & EC & pH & FE $(\%)$ \\
\hline \multicolumn{14}{|l|}{ TW } \\
\hline SL & $0.753^{* *}$ & & & & & & & & & & & & \\
\hline SG & $0.702^{* *}$ & $0.924^{* *}$ & & & & & & & & & & & \\
\hline SFW & $0.595^{*}$ & $0.829^{* *}$ & $0.822^{* *}$ & & & & & & & & & & \\
\hline SDW & $0.704^{* *}$ & $0.911^{* *}$ & $0.927^{* *}$ & $0.832^{* *}$ & & & & & & & & & \\
\hline VI-I & $0.741^{* *}$ & $0.973^{* *}$ & $0.987^{* *}$ & $0.839^{* *}$ & $0.935^{* *}$ & & & & & & & & \\
\hline VI-II & $0.735^{* *}$ & $0.948^{* *}$ & $0.934^{* *}$ & $0.843^{* *}$ & $0.994^{* *}$ & $0.956^{* *}$ & & & & & & & \\
\hline $\mathrm{TZ}$ & $0.713^{* *}$ & $0.964^{* * *}$ & $0.945^{* *}$ & $0.780^{* *}$ & $0.880^{* *}$ & $0.970^{* * *}$ & $0.911^{* *}$ & & & & & & \\
\hline AAT & $0.749^{* * *}$ & $0.758^{* *}$ & $0.647^{* *}$ & $0.576^{*}$ & $0.524^{*}$ & $0.712^{* * *}$ & $0.590^{*}$ & $0.743^{* *}$ & & & & & \\
\hline DHA & $0.814^{* *}$ & $0.867^{* *}$ & $0.861^{* *}$ & $0.668^{* *}$ & $0.806^{* *}$ & $0.882^{* *}$ & $0.831^{* *}$ & $0.885^{* *}$ & $0.777^{* * *}$ & & & & \\
\hline EC & $-0.518^{*}$ & $-0.896^{* * *}$ & $-0.824^{* *}$ & $-0.860^{* *}$ & $-0.812^{* *}$ & $-0.871^{* *}$ & $-0.844^{* *}$ & $-0.863^{* * *}$ & $-0.677^{* *}$ & $-0.742^{* *}$ & & & \\
\hline $\mathrm{pH}$ & $0.701^{* *}$ & $0.879^{* * *}$ & $0.810^{* *}$ & $0.858^{* *}$ & $0.808^{* * *}$ & $0.851^{* * *}$ & $0.842^{* *}$ & $0.831^{* * *}$ & $0.747^{* *}$ & $0.796^{* *}$ & $-0.800^{* *}$ & & \\
\hline $\mathrm{FE}(\%)$ & $0.653^{* *}$ & $0.839^{* *}$ & $0.879^{* *}$ & $0.808^{* *}$ & $0.742^{* *}$ & $0.880^{* *}$ & $0.772^{* *}$ & $0.890^{* *}$ & $0.729^{* *}$ & $0.757^{* *}$ & $-0.845^{* *}$ & $0.734^{* *}$ & \\
\hline
\end{tabular}

*Significant at $5 \%(\mathrm{p}=0.05)$

$* *$ Significant at $1 \%(\mathrm{p}=0.01)$

TW = Test Weight, SL = Seedling length, SG =standard germination, SFW = Seedling fresh weight, SDW = Seedling dry weight, VI-I = Vigour index-I, VI-II = Vigour index-II, TZ = Tetrazolium test, AA = Accelerated ageing test, DHA = Dehydrogenase activity test, EC =

Electrical conductivity test, $\mathrm{pH}=\mathrm{pH}$ exudates test, $\mathrm{FE}=$ Field emergence. 
The correlations between field and laboratory parameters of one year old seed lot are presented in Table 2. Field emergence showed positive and significant correlation with test weight (0.795), seedling length (0.804), standard germination (0.805), seedling fresh weight (0.739), seedling dry weight (0.819), vigour index- I (0.850), vigour index-II (0.837), tetrazolium test $(0.834)$, accelerated ageing test (0.786), dehydrogenase activity test $(0.720)$ and $\mathrm{pH}$ exudates (0.706) while negative and significant correlation was found with electrical conductivity (0.729). Standard germination showed positive and significant correlation with test weight $(0.750)$, seedling length (0.794), seedling fresh weight (0.739), vigour index-I (0.907), vigour index-II (0.931), tetrazolium test $(0.931)$, accelerated ageing test (0.607), dehydrogenase activity test $(0.747), \mathrm{pH}$ exudates test $(0.772)$ and field emergence $(0.805)$ while negatively and significant correlation was found with electrical conductivity (0.787). Shridhar and Nagaraja (2004) reported that the standard germination test in cotton had a positive significant correlation with field emergence. Kuklik and Yaklich (1982) and Egli and Tekrony (1985) observed that standard germination and AA tests were significantly correlated with field emergence in soybean Similar results were also reported in coriander (Deshraj, 2002) and in indian mustard (Punia et al., 2006).

The correlations between field and laboratory parameters of two year old seed lot are presented in Table 3. Field emergence showed positive and significant correlation with seedling length (0.570), standard germination (0.540), vigour index-I (0.603), vigour indexII (0.553), tetrazolium test (0.623) and accelerated ageing test (0.526). Yadav and Dhankar (2001) reported that vigour indices were positively and significantly correlated with standard germination, seedling length and seedling dry weight and negatively correlated with electrical conductivity in okra. Above results are in agreement with various workers in different crops such as fennel (Mor et al., 2009) and in coriander (Kumar, 2007).

The correlation between field and laboratory parameters of means of naturally aged seed of fennel is presented in Table 4. Standard germination is positively and significantly correlated with vigour index-I (0.987), vigour index-II (0.934), tetrazolium test (0.945), accelerated ageing test (0.647), dehydrogenase activity test $(0.861), \mathrm{pH}$ exudates test $(0.810)$ and field emergence (0.879) while negatively and significantly correlated with electrical conductivity (0.824). Field emergence was positively and significantly correlated with seedling length (0.839), standard germination (0.879), seedling fresh weight (0.808), seedling dry weight (0.742), vigour index-I (0.880), vigour index-II (0.772), tetrazolium test $(0.890)$, accelerated ageing test $(0.729)$, dehydrogenase activity test $(0.757), \mathrm{pH}$ exudates test (0.734) while negatively and significantly correlated with electrical conductivity (0.845). As standard germination, seedling vigour index-I and tetrazolium test are highly correlate with field emergence they can be used as reliable predictors of field emergence (\%). Usha (2009) reported that standard germination test is the best indicator of seed vigour in onion, as highest correlations $(\mathrm{P}<0.01)$ were obtained between initial germination $(\%)$ and field emergence $(r=0.937 * *)$. The present results are also in corroborate with the findings of Kumar et al., 2015 in coriander, Kumar, 2004 in onion and Sadik, 2012 in ajwain.

In conclusion, present study revealed that different seed quality parameters were positively and significantly correlated with field emergence except test weight while electrical conductivity was negatively and 
significantly correlated with field emergence. As standard germination, tetrazolium test and dehydrogenase activity test were highly associated with field emergence, they can be used as reliable predictors of field emergence $(\%)$.

\section{References}

Barros, L., Carvalho, A.M. and Ferreira, I.C.F.R. 2010. The nutritional composition of fennel (Foeniculum vulgare): shoots, leaves, stems and inflorescences. LWT: Food Sci. Technol., 43(5): 814-818.

Deshraj. 2002. Studies on viability and vigour in coriander (Coriandrum sativum L.). M.Sc. Thesis submitted to CCS HAU, Hisar

Egli, D.B. and Tekrony, D.M. 1985. Soybean seed germination vigour and field emergence. Seed Sci. and Tech., 23(3): 595 - 607.

Kuklik, M.M. and Yaklich, R.W. 1982. Evaluation of vigour tests in soybean seeds Relationship of accelerated ageing, cold test, sand bench, and speed of germination tests to field performance. Crop Sci., 22: 766-770.

Kumar, A. 2004. Seed quality assessment in naturally aged seed of onion (Allium cepa). M.Sc. Thesis submitted to CCS Haryana Agricultural University, Hisar.

Kumar, A. 2007. Seed quality assessment in naturally aged seeds of coriander (Coriandrum sativum L.). M. Sc. Thesis submitted to CCS Haryana Agricultural University, Hisar

Kumar, V., Verma, S.S. and Verma, U. and Kumar, A. 2015. Seed viability and vigour in naturally aged seeds of coriander (Coriandrum sativum). Indian J. Agric. Sci., 85(4): 561-5

Kumari, S., Kumar, A. and Tehlan, S.K. 2014.
Studies on physiological parameters in fenugreek under ambient conditions. Annals of Biol. 30(4): 691-695

Mor, V.S., Verma, S.S., and Verma, U. 2009. studies on seed viability and vigor in various order. Seed Sci. \& Technol., 37: 747-757

Ostle, B. and Mensing, R.W. 1975. Status in research. In: Basic concepts and techniques for Research workers ed. $\left(3^{\text {rd }}\right)$. The IOWA state University Press/Ames. Oxford and IBH Pub. Co.

Perry, D.A. 1978. Report of vigour test committee 1974-1977, Seed Sci. Technol., 6: 159-181.

Punia, R.C., Dahiya, O.S. and Kumar, A. 2006. Exudate $\mathrm{pH}$ test: A quick and reliable predictor of seedling establishment in India Mustard [Brassica juncea (L.) Czern and Coss]. Natnl. J. Pl. Improv., 8 (2): 103 105

Ram, C., Singh, O., Kharab, R.P.S., Kumari, P. and Yadava, T.P. 1991. Seedling vigour in pigeonpea. Seed Sci. and Tech., 19: 627631.

Sadik, M. 2012. Studies on seed viability and vigour in Ajwain (Trachyspermum copticum L.). M. Sc. Thesis, submitted to CCS Haryana Agricultural University, Hisar.

Shridhar, G. and Nagaraja, A. 2004. Studies on relationship between vigour tests and field emergence. Mysore J. Agric. Sci., 38(4): 551-553.

Usha, T. N. 2009. Evaluation and enhancement of seed vigour in onion and soybean. Ph.D. Thesis, IARI, New Delhi.

Yadav, S K. and Dhankar, B.S. 2001. Correlation studies between various fields parameters and seed quality traits in okra cv. Versha uphar. Seed Res., 29(1): 84-88.

\section{How to cite this article:}

Deepak Kumar Singhal, Vinod Kumar, S.K. Tehlan, Pooja Rani and Amit Kumar. 2017. Interrelationship between Different Seed Quality Parameters in Fennel Int.J.Curr.Microbiol.App.Sci. 6(5): 553-560. doi: https://doi.org/10.20546/ijcmas.2017.605.064 